A NOVEL FOUR NODE Q UADRILATERAL SMOOTH ING ELEMENT FOR STRESS ENHANCEMENT AND ERROR ESTIMATION*

A. Tessler

Computational Structures Branch

NASA Langley Research Center Hampton, VA 23681-0001
H. R. Riggs

Civil Engineering Dept.

University of Hawaii at Manoa

Honolulu, HI 96822
M. Dambach

George Washington University

NASA Langley Research Center

Hampton, VA 23681-0001

\section{A bstract}

A four-node, quadrilateral smoothing element is developed based upon a penalized-discrete-least-squares variational formulation. The smoothing methodology recovers $\mathrm{C}^{1}$-continuous stresses, thus enabling effective a posteriori error estimation and automatic adaptive mesh refinement. The element formulation is originated with a five-node macro-element configuration consisting of four triangular anisoparametric smoothing elements in a cross-diagonal pattern. This element pattern enables a convenient closed-form solution for the degrees of freedom of the interior node, resulting from enforcing explicitly a set of natural edge-wise penalty constraints. The degree-of-freedom reduction scheme leads to a very efficient formulation of a four-node quadrilateral smoothing element without any compromise in robustness and accuracy of the smoothing analysis. The application examples include stress recovery and error estimation in adaptive mesh refinement solutions for an elasticity problem and an aerospace structural component.

\section{In troduction}

The displacement-based finite element method has become a standard tool for structural analysis. However, due to its inherent interelement stress discontinuity, it generally requires a special post-processing or 'recovery' procedure to enable improved stress calculations. The improved stress predictions can further be utilized in a posteriori error estimation that in turn enables automatic mesh adaptivity. The recovery procedures developed to date can be classified as (a) local (i.e., element-level), (b) patch-based, or (c) global. The simple averaging of nodal stresses from adjacent elements is an example of the simplest patch-based stress recovery. Early attempts to employ global leastsquares procedures to obtain continuous stress fields

\footnotetext{
* Copyright (C) 1998 by the American Institute of Aeronautics and Astronautics, Inc. No copyright is asserted in the United States under title 17, U.S. Code. The U.S. Government has a royalty-free license to exercise all rights under the copyright claimed herein for Governmental purposes. All rights are reserved by the copyright owner.
}

were not widely adopted. ${ }^{1,2}$ Instead, local element-level least-squares approaches appeared to be more attractive because of their intrinsic computational efficiency. ${ }^{2,3}$ However, the local schemes do not recover a continuous stress field, and a subsequent nodal averaging is generally used to achieve the stress continuity along element interfaces. With the recognition that certain interior stresses exhibit superconvergent properties, ${ }^{4}$ there evolved a class of local procedures involving 'projection' of these stresses to the element boundary, although other local procedures were also proposed. ${ }^{5}$

Recent developments in stress recovery procedures have largely been motivated by a posteriori error estimation, ${ }^{6}$ especially for use in automatic adaptive mesh refinement. In this context, polynomial patch recovery procedures have been explored. Zienkiewicz and $\mathrm{Zhu}^{7,8}$ developed the Superconvergent Patch Recovery (SPR) procedure, and various modifications of their approach have been proposed subsequently. ${ }^{9-11}$ These procedures generally attempt to recover from the superconvergent stress points a stress field with superconvergent properties. The latest formulation by Boroomand and Zienkiewicz ${ }^{12,13}$ explored yet another polynomial patch-based procedure which uses nodal equilibrium rather than superconvergent stresses. In general, patch procedures are used to recover the stresses at nodes. If a node belongs to multiple patches, an averaging procedure must be used. The continuous stress field is then defined in an ad hoc manner by the nodal stresses and the finite element displacement interpolation functions.

A major departure from other procedures is the finite element-based recovery methodology of Tessler and coworkers. ${ }^{14-21}$ The methodology, which is based on the minimization of a Penalized-Discrete-Least-Squares (PDLS) error functional, is highly effective and is designed to provide improved stress predictions with a higher degree of smoothness and to obtain robust a posteriori error estimates.

The error functional involves a discrete least-squares term in which discrete finite element stresses are compared with continuous recovered stresses, a penalty constraint term that enforces $\mathrm{C}^{1}$-continuity of the recovered stresses, and a curvature-control term that ensures stability and robustness of the method. The post- 
processing is performed via the Smoothing Element Analysis (SEA) which itself is a finite element method. As such, SEA has its own mesh and element-based interpolation functions which minimize the PDLS error functional. The methodology is capable of recovering superconvergent stresses of higher accuracy and continuity than the original finite element stresses. The approach is unique in that the recovered stress field is essentially $\mathrm{C}^{1}$-continuous. In addition to achieving a higher degree of continuity, SEA generally produces more accurate stresses than the SPR method. The smoothed stresses can be used for design since they represent more accurate stresses than the ones obtained directly from a finite element analysis. They can also be employed in a posteriori error estimators. Importantly, SEA fits perfectly in any general purpose finite element code since it has the same architecture as the standard finite element method. ${ }^{22-24}$

The smoothing element developed by Tessler and coworkers is a three-node triangle, incorporating a quadratic interpolation for the stress, and linear interpolations for the two normal stress derivatives. ${ }^{20,21}$ Since the PDLS error functional possesses a penaltyconstraint term, the use of anisoparametric interpolations is key to enforcing pointwise $\mathrm{C}^{1}$ stress continuity without developing the detrimental locking effect. The method is especially robust and effective when the smoothing element discretization is represented by quadrilaterals consisting of four triangular smoothing elements in a cross-diagonal pattern. The resulting macro-element consists of five nodes, each having three degrees-of-freedom (dof's).

In this paper, a computationally efficient, four-node quadrilateral smoothing element is developed. The element is derived from a five-node macro-element consisting of four triangular smoothing elements in a cross-diagonal pattern. This element pattern enables an exact closed-form solution for the dof's of the interior node, resulting from enforcing explicitly a set of natural edge-wise penalty constraints. The degree-of-freedom reduction scheme gives rise to a four-node quadrilateral smoothing element which has obvious computational advantages over the five-node macro-element. In particular, its mesh results in nearly one-half the dof's than would a corresponding cross-diagonal mesh constructed with triangular elements. Because an exact closed-form solution is used for the reduction, no compromise is made in terms of robustness and accuracy by reducing the dof's.

The conceptual framework and variational basis of SEA/PDLS are first discussed. Then, smoothing equations for a triangular element are derived on the basis of consistent anisoparametric interpolations for the stress and two normal stress derivatives. Eight edge-wise constraint equations are formulated for a five-node macro-element consisting of four triangles in a crossdiagonal pattern. Three of these equations are solved in closed form for the dof's corresponding to the interior node. The resulting transformation relations are used to formulate the four-node smoothing element in a computationally efficient manner. Numerical examples include stress recovery and error estimation in adaptive mesh refinement solutions for an elasticity problem and an aerospace structural component.

\section{$\underline{\text { SE A C on ceptual F ram ew ork }}$}

The basic conceptual framework of the present stress recovery and error estimation approach is illustrated in Fig. 1. Based upon a Finite Element Analysis (FEA), which can be quite general and involve either linear or nonlinear solution procedures, a subsequent Smoothing Element Analysis (SEA) is carried out which discretizes the same physical domain of the problem. The SEA employs the discrete FEA stresses $\sigma^{h}$ as the prescribed input data, using either Gauss integration point stresses or superconvergent stresses. These stress locations are preferred because of their superior accuracy compared to other stresses. Alternatively, quantities other than stress components can also be smoothed in the same manner, for example, discrete displacement data, ${ }^{14}$ strain measures, ${ }^{19}$ stress invariants, element strain energies, or any other discrete data of interest.

The SEA itself is a finite element method and requires its own mesh which in general can differ from the finite element analysis mesh. Thus, the problem domain $\Omega$ is discretized with $\mathrm{n}_{\mathrm{el}}$ smoothing finite elements such that $\Omega=\cup_{\mathrm{e}=1}^{\mathrm{n}_{\mathrm{el}}} \Omega^{\mathrm{e}}$, where $\Omega^{\mathrm{e}}$ is the domain of a smoothing element. Within each smoothing element, a penalized-discrete-least-squares (PDLS) error functional is formulated and is based upon a smooth stress $\sigma^{\mathrm{s}}$ and two stress-gradient variables. These field variables are interpolated with compatible shape functions that ensure a practically $\mathrm{C}^{1}$-continuous stress field and $\mathrm{C}^{0}$-continuous stress gradients. The total PDLS error functional contributed by all smoothing elements in the discretization is minimized with respect to all dof's of the smoothing field variables. The resulting smoothing equations, which have a standard linear algebraic form, produce an improved and pointwise smooth stress field. The recovered smooth stress field can either be used directly for design calculations or they can be employed for a posteriori error estimation in an automatic adaptive refinement process, which is perhaps of greater significance. 

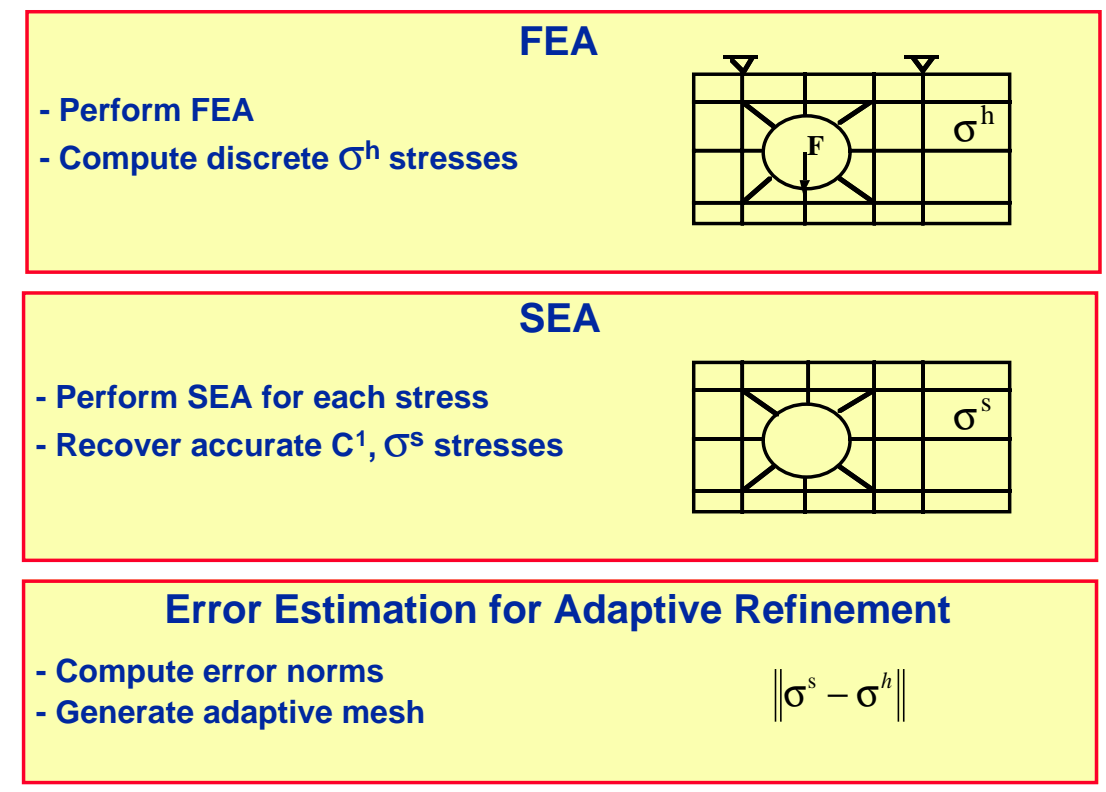

Fig. 1. Smoothing element analysis for stress recovery and error estimation.

In developing a practical and theoretically appropriate framework for the application of SEA to built-up structural models, such as those found in aerospace and automotive structures, it is important to realize that stresses belonging to different structural components may, in fact, be physically discontinuous where such components intersect. Moreover, in most cases, stress components in one shell or beam segment would lack the same definition in another segment. For these reasons, it may not be meaningful to employ a scheme which would require continuity of stresses along structural interfaces. Also, the usual averaging of stresses along structural interfaces would often result in erroneous stress results at the given interface and the surrounding region.

The application of SEA to built-up structures is undertaken herein by associating individual structural segments with an independent SEA mesh. This approach should be applicable to one-dimensional (beams and trusses), two-dimensional (plates and shells), and three-dimensional (solid element models) structural finite element approximations. Thus the SEA discretization would consist of distinct domainbased meshes, each totally independent of the adjacent SEA domain. The SEA domain-based framework will be further illustrated on a built-up aerospace structure discussed in the Numerical Results section.

\section{Error Functional}

The mathematical foundation of SEA is described for a stress field defined on a flat two-dimensional domain,
$\Omega=\left\{\mathbf{x} \in \mathfrak{R}^{2}\right\}$, for which $\mathbf{x}=\{\mathrm{x}, \mathrm{y}\}$ denotes the position vector in Cartesian coordinates. As will be shown subsequently, this two-dimensional framework can be readily extended for application to typical builtup aerospace shell structures.

For a single smoothing element within a SEA mesh, the PDLS error functional may be expressed for a given stress component $\sigma$ as

$$
\begin{aligned}
& \Phi^{\mathrm{e}}=\frac{1}{\mathrm{~N}} \sum_{\mathrm{q}=1}^{\mathrm{n}^{\mathrm{e}}}\left[\sigma_{\mathrm{q}}^{\mathrm{h}}-\sigma^{\mathrm{s}}\left(\mathbf{x}_{\mathrm{q}}\right)\right]^{2}+ \\
& \alpha \sum_{\mathrm{e}=1}^{\mathrm{n}^{\mathrm{e}}} \int_{\Omega^{\mathrm{e}}}\left[\left(\sigma_{, \mathrm{x}}^{\mathrm{s}}-\theta_{\mathrm{x}}^{\mathrm{s}}\right)^{2}+\left(\sigma_{, \mathrm{y}}^{\mathrm{s}}-\theta_{\mathrm{y}}^{\mathrm{s}}\right)^{2}\right] \mathrm{d} \Omega+ \\
& \beta \sum_{\mathrm{e}=1}^{\mathrm{n}^{\mathrm{e}}} \Omega^{\mathrm{e}} \int_{\Omega^{\mathrm{e}}}\left[\left(\theta_{\mathrm{x}, \mathrm{x}}^{\mathrm{s}}\right)^{2}+\left(\theta_{\mathrm{y}, \mathrm{y}}^{\mathrm{s}}\right)^{2}+\right. \\
& \left.\frac{1}{2}\left(\theta_{\mathrm{x}, \mathrm{y}}^{\mathrm{s}}+\theta_{\mathrm{y}, \mathrm{x}}^{\mathrm{s}}\right)^{2}\right] \mathrm{d} \Omega
\end{aligned}
$$

where, for simplicity, the usual tensorial subscripts for the stress are omitted; a comma denotes partial differentiation, $\alpha$ and $\beta$ are dimensionless parameters, and $\mathrm{N}$ denotes the total number of sampled discrete stresses. For smoothing purposes, $\sigma$ is treated as a scalar quantity. As will be demonstrated shortly, the variables $\theta_{\mathrm{i}}^{\mathrm{S}}(\mathrm{i}=\mathrm{x}, \mathrm{y})$ represent the first-order partial derivatives of $\sigma^{\mathrm{s}}$ with respect to the spatial coordinates. The finite element stress field is evaluated (or sampled) in each element at $\mathbf{x}_{\mathrm{q}}\left(\mathrm{q}=1,2, \ldots, \mathrm{n}^{\mathrm{e}}\right)$, to obtain the 
set of discrete stresses $\left\{\sigma_{\mathrm{q}}^{\mathrm{h}}\right\}$, i.e., $\sigma_{\mathrm{q}}^{\mathrm{h}} \equiv \sigma^{\mathrm{h}}\left(\mathbf{x}_{\mathrm{q}}\right)$, and where $\mathrm{n}^{\mathrm{e}}$ is the number of the sampled stresses within the element. Because the highest partial derivative in (1) is of order one, the field variables need only be approximated with $\mathrm{C}^{0}$-continuous shape functions. The functional is minimized with respect to all nodal dof's and yields a system of linear algebraic equations which must be solved for the dof's.

The first term in (1) is a normalized discrete leastsquares functional in which the squared 'error' between the smoothed stress field and the sampled stress data is computed for all sampled stresses. The second term in (1) represents a penalty functional which, for sufficiently large $\alpha$, enforces the derivatives of the smoothed stress field $\sigma_{, i}^{\mathrm{s}}$ to approach the corresponding $\theta_{\mathrm{i}}^{\mathrm{s}}$ variables pointwise, i.e.,

$$
\sigma_{, i}^{\mathrm{s}} \rightarrow \theta_{\mathrm{i}}^{\mathrm{s}} \quad(\mathrm{i}=\mathrm{x}, \mathrm{y}) \text { in } \Omega^{\mathrm{e}} .
$$

The greater the value of $\alpha$, the closer the correlation between $\sigma_{, i}^{\mathrm{s}}$ and $\theta_{\mathrm{i}}^{\mathrm{s}}$, where $C^{1}$ continuity of $\sigma^{\mathrm{s}}$ is achieved as $\alpha \rightarrow \infty$. For the practical application of the method, $\alpha$ needs to be sufficiently large in order to enforce conditions (2); however, it should not be excessively large to cause ill-conditioning of the smooth solution. The range $\alpha=10^{2}-10^{6}$ has been demonstrated in previous studies to be quite adequate for these purposes. Because $\theta_{\mathrm{i}}^{\mathrm{s}}$ are interpolated with continuous functions, the smoothed stress field, for all practical purposes, can achieve $\mathrm{C}^{1}$ continuity throughout the $\Omega$ domain.

The third term in (1) involves squared first derivatives of the $\theta_{\mathrm{i}}^{\mathrm{s}}$ variables which, in accordance with (2), represent the curvatures (i.e., second partial derivatives) of the smoothed stress field in the limiting sense. (i.e., $\sigma_{, i j}^{\mathrm{s}} \rightarrow \theta_{\mathrm{i}, \mathrm{j}}^{\mathrm{s}}$ ) For the shape functions subsequently considered for the smoothing element, the $\theta_{\mathrm{i}, \mathrm{j}}^{\mathrm{s}}$ terms represent the curvatures of the smoothed stress exactly. The third term imposes a constraint condition on the stress-curvature field, the severity of which is governed by the value of the parameter $\beta$. When the sampled stress data is perceived to be reasonably accurate, the $\beta$ parameter needs to be very small, particularly in relation to the penalty parameter $\alpha$. For the sampled stress data exhibiting substantial error, larger values of $\beta$ need to be used to smooth or filter the data. A more general form of (1) which enables the use of appropriate weighting functions for the first and second terms can be found in Ref. 20.
A notable observation regarding the functional form of the penalty-constraint and curvature-control terms in (1) is that they can be seen as mathematical analogs of the transverse shear and bending energies in ReissnerMindlin plate theory. In this connection, successful element technologies developed for plate elements can equally be applied in this formulation. ${ }^{25-27}$

\section{Sm ooth ing E lem ent In terp olation s}

The description of a convenient and effective interpolation strategy for the field variables to be approximated in (1) is addressed. Although the functional in (1) admits $\mathrm{C}^{0}$-continuous shape functions for the field variables $\sigma^{\mathrm{s}}, \theta_{\mathrm{x}}^{\mathrm{s}}$, and $\theta_{\mathrm{y}}^{\mathrm{s}}$, the constraints in (2) severely limit the suitable choice of the shape functions. The reasons for these restrictions lie in the character of these constraint equations. Unless appropriate interpolations are selected for the field variables, the resulting solution will suffer from 'locking'. In the context of the smoothing analysis, 'locking' would be manifested by the smoothed solution that grossly underestimates the stress field and is thus rendered to be useless. The locking effect and the computational means of avoiding it have been studied extensively, especially in the context of shear-deformable bending finite elements. ${ }^{25-27}$ Although the earlier approaches favored reduced integration of the penalty term (a counterpart of the second term in (1)), the improved understanding of this phenomenon in recent years seems to favor the use of special interpolation schemes that ensure consistency both in the constraint and variational sense. Such interpolation approaches, interchangeably called interdependent and anisoparametric, were originally introduced by Tessler and co-workers ${ }^{26-28}$ in their sheardeformable beam and plate formulations.

The three-node triangular smoothing element employs the anisoparametric interpolations of the lowest order, that is $\sigma^{\mathrm{s}}$ is interpolated with a complete quadratic polynomial and the $\theta_{\mathrm{x}}^{\mathrm{s}}$ and $\theta_{\mathrm{y}}^{\mathrm{s}}$ employ linear interpolations. In reference to the notation in Fig. 2, these smoothing element interpolations may be expressed in matrix form as :

$$
\begin{gathered}
\sigma^{\mathrm{s}}=\mathbf{P s}+\mathbf{Q}_{\mathrm{x}} \mathbf{s}_{\mathrm{x}}+\mathbf{Q}_{\mathrm{y}} \mathbf{s}_{\mathrm{y}} \equiv \mathbf{N} \mathbf{d}^{\mathrm{e}}, \\
\theta_{\mathrm{x}}^{\mathrm{s}}=\mathbf{P s}_{\mathrm{x}}, \quad \theta_{\mathrm{y}}^{\mathrm{s}}=\mathbf{P s}_{\mathrm{y}}
\end{gathered}
$$

where $\mathbf{s}=\left[\sigma_{1}, \sigma_{2}, \sigma_{3}\right]^{\mathrm{T}}, \mathbf{s}_{\mathrm{x}}=\left[\theta_{\mathrm{x} 1}, \theta_{\mathrm{x} 2}, \theta_{\mathrm{x} 3}\right]^{\mathrm{T}}$, and $\mathbf{s}_{\mathrm{y}}=\left[\theta_{\mathrm{y} 1}, \theta_{\mathrm{y} 2}, \theta_{\mathrm{y} 3}\right]^{\mathrm{T}}$ are the vectors of nodal dof's, and $\mathbf{P}, \mathbf{Q}_{\mathrm{x}}$, and $\mathbf{Q}_{\mathrm{y}}$ are the row-vectors of linear and quadratic shape functions, respectively. The inter- 
polation functions given in terms of triangular areaparametric coordinates, $\mathbf{z}=\left[\zeta_{1}, \zeta_{2}, \zeta_{3}\right]$, can be written as

$$
\begin{aligned}
\mathrm{P}_{\mathrm{i}}=\zeta_{\mathrm{i}}, \mathrm{Q}_{\mathrm{xi}} & =\frac{1}{2}\left(\mathrm{a}_{\mathrm{k}} \zeta_{\mathrm{i}} \zeta_{\mathrm{j}}-\mathrm{a}_{\mathrm{j}} \zeta_{\mathrm{i}} \zeta_{\mathrm{k}}\right), \\
\mathrm{Q}_{\mathrm{yi}} & =\frac{1}{2}\left(\mathrm{~b}_{\mathrm{j}} \zeta_{\mathrm{i}} \zeta_{\mathrm{k}}-\mathrm{b}_{\mathrm{k}} \zeta_{\mathrm{i}} \zeta_{\mathrm{j}}\right)
\end{aligned}
$$

in which

$$
\begin{gathered}
\zeta_{i}=\frac{c_{i}+b_{i} x+a_{i} y}{2 A}, \quad a_{i}=x_{k}-x_{j}, \quad b_{i}=y_{j}-y_{k}, \\
c_{i}=x_{j} y_{k}-x_{k} y_{j}, \quad A=\frac{1}{2}\left(a_{3} b_{2}-a_{2} b_{3}\right),
\end{gathered}
$$

with A representing the area of a triangular element, and $\mathrm{x}_{\mathrm{k}}, \mathrm{y}_{\mathrm{k}}$ the nodal Cartesian coordinates; in the foregoing equations, the subscripts are given by the cyclic permutation of $\mathrm{i}=1,2,3, \mathrm{j}=2,3,1$, and $\mathrm{k}=3,1$, 2. Note that these interpolations are consistent with a three-node element which has only three dof's per node.

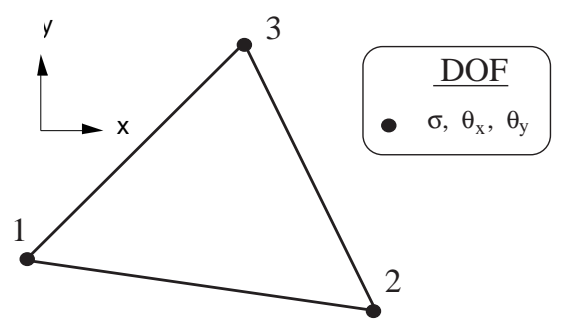

Fig. 2. Notation for three-node anisoparametric smoothing element.

The following attributes delineate the most notable benefits of the three-node anisoparametric triangular element for utilization in SEA: (a) The element interpolations (3) and (4) ensure that the gradient of the smoothed stress, $\sigma_{, i}^{\mathrm{s}}$, is the same degree polynomial as that representing $\theta_{\mathrm{i}}^{\mathrm{s}}$, i.e., they are both linearly distributed across $\Omega^{\mathrm{e}}$; this interpolational consistency is the key to the proper element-level resolution of constraints (2), (b) The triangular element permits a one-to-one linear mapping between the global and element (area-parametric) coordinates, thus allowing a straightforward mapping of the sampled stress data onto the smoothing element, and (c) The quadratic distribution of $\sigma^{\mathrm{s}}$ is relatively low order and thus ensures sufficient robustness of the method both in terms of interpolation and extrapolation.

\section{$\underline{\text { Sm ooth ing Equation S }}$}

The element smoothing equations are obtained by introducing (3) into (1) and taking the first variation with respect to the nodal dof's (i.e., $\delta \Phi^{\mathrm{e}}=0$ ), giving rise to

$$
\mathbf{K}^{\mathrm{e}} \mathbf{d}^{\mathrm{e}}=\mathbf{F}^{\mathrm{e}}
$$

where $\mathbf{d}^{\mathrm{e}}$ is a vector containing the element nodal dof's, $\mathbf{K}^{\mathrm{e}}$ is a symmetric, element smoothing matrix, and $\mathbf{F}^{\mathrm{e}}$ is a consistent right-hand-side vector. The element matrices are given as

$$
\begin{aligned}
\mathbf{K}^{\mathrm{e}} & \equiv \mathbf{K}_{\varepsilon}^{\mathrm{e}}+\mathbf{K}_{\alpha}^{\mathrm{e}}+\mathbf{K}_{\beta}^{\mathrm{e}} \\
& =\frac{1}{\mathrm{~N}} \sum_{\mathrm{q}=1}^{\mathrm{n}^{\mathrm{e}}} \mathbf{N}_{\mathrm{q}}^{\mathrm{T}} \mathbf{N}_{\mathrm{q}}+\alpha \int_{\Omega^{\mathrm{e}}} \mathbf{B}_{\alpha}^{\mathrm{T}} \mathbf{B}_{\alpha} \mathrm{d} \Omega+ \\
& \beta \Omega^{\mathrm{e}} \int_{\Omega^{\mathrm{e}}} \mathbf{B}_{\beta}^{\mathrm{T}} \mathbf{D} \mathbf{B}_{\beta} \mathrm{d} \Omega, \\
\mathbf{F}^{\mathrm{e}} & =\frac{1}{\mathrm{~N}} \sum_{\mathrm{q}=1}^{\mathrm{n}^{\mathrm{e}}} \sigma_{\mathrm{q}}^{\mathrm{h}} \mathbf{N}_{\mathrm{q}}^{\mathrm{T}}, \quad \mathbf{d}^{\mathrm{e}}=\left\{\begin{array}{c}
\mathbf{s} \\
\mathbf{s}_{\mathrm{x}} \\
\mathbf{s}_{\mathrm{y}}
\end{array}\right\}
\end{aligned}
$$

and

$$
\begin{gathered}
\mathbf{B}_{\alpha}=\left[\begin{array}{ccc}
\mathbf{P}_{, \mathrm{x}} & \mathbf{Q}_{\mathrm{x}, \mathrm{x}}-\mathbf{P} & \mathbf{Q}_{\mathrm{y}, \mathrm{x}} \\
\mathbf{P}_{, \mathrm{y}} & \mathbf{Q}_{\mathrm{x}, \mathrm{y}} & \mathbf{Q}_{\mathrm{y}, \mathrm{y}}-\mathbf{P}
\end{array}\right], \\
\mathbf{B}_{\beta}=\left[\begin{array}{ccc}
\mathbf{0} & \mathbf{P}_{, \mathrm{x}} & \mathbf{0} \\
\mathbf{0} & \mathbf{0} & \mathbf{P}_{, \mathrm{y}} \\
\mathbf{0} & \mathbf{P}_{, \mathrm{y}} & \mathbf{P}_{, \mathrm{x}}
\end{array}\right], \quad \mathbf{D}=\left[\begin{array}{ccc}
1 & 0 & 0 \\
0 & 1 & 0 \\
0 & 0 & \frac{1}{2}
\end{array}\right]
\end{gathered}
$$

where the element matrix $\mathbf{K}^{\mathrm{e}}$ is comprised of the error matrix, $\mathbf{K}_{\varepsilon}^{\mathrm{e}}$, the penalty-constraint matrix, $\mathbf{K}_{\alpha}^{\mathrm{e}}$, and the curvature-control matrix, $\mathbf{K}_{\beta}^{\mathrm{e}}$; and $\mathbf{N}_{\mathrm{q}} \equiv \mathbf{N}\left(\mathbf{x}_{\mathrm{q}}\right)$. Equation (6) is integrated using exact integration formulas. Note that integration of $\mathbf{K}_{\beta}^{\mathrm{e}}$ is trivial since its integrand is constant.

The global smoothing equations, $\mathbf{K d}=\mathbf{F}$, are obtained using the usual finite element assembly operation and are solved for each stress component. Conveniently, $\mathbf{K}^{\mathrm{e}}$ depends only on the element shape functions that are evaluated at the sampled stress locations and does not involve the stress values. This means that the smoothing of an individual stress component corresponds to a different right-hand side only, stored in the $\mathbf{F}$ vector. Thus, regardless of the number of smoothed quantities, the global $\mathbf{K}$ matrix need only be assembled and factored once. Employing a very small $\beta$ parameter (say, $10^{-5}$ ) assures that $\mathbf{K}$ is nonsingular even when the minimal number of sampling stresses, $\mathrm{N}=3$, is available. ${ }^{17}$ Although this aspect permits a great range of possibilities for constructing a SEA mesh, it is highly desirable for 
automation purposes that the SEA mesh be identical to the FEA mesh.

\section{Four-N ode Q uadrilateral via E dge Constrain ts}

The three-node triangular interpolations described in the previous section are particularly effective and robust when the smoothing element discretization is locally represented by quadrilaterals consisting of four triangular smoothing elements in a cross-diagonal pattern. ${ }^{20,21}$ The resulting macro-element has five nodes, and a total of 15 dof's. Such a macro-element, in which large penalty numbers ensure virtually exact satisfaction of the constraints (2), can be used without causing any locking-type deterioration, or even degradation in accuracy.

Once (3) are introduced into (2), a straightforward manipulation of the resulting equations produces three edge-wise constraints per element. It then follows that the five-node macro-element (refer to Fig. 3) would possess a total of eight such edge constraint equations four interior and four exterior. Replacing the limiting condition with that of equality in (2), which for large values of $\alpha$ should be valid for all practical purposes, the edge constraint equations can be written in the form:

\section{In terior $\mathrm{E}$ dge $\mathrm{C}$ on stra in ts}

$$
\begin{gathered}
\sigma_{\mathrm{i}}-\sigma_{5}=\mathrm{a}_{\mathrm{i} 5}\left(\theta_{\mathrm{xi}}+\theta_{\mathrm{x} 5}\right)+\mathrm{b}_{\mathrm{i} 5}\left(\theta_{\mathrm{yi}}+\theta_{\mathrm{y} 5}\right), \\
\mathrm{i}=1,2,3,4
\end{gathered}
$$

where $a_{i 5}=\frac{1}{2}\left(x_{i}-x_{5}\right), \quad b_{i 5}=\frac{1}{2}\left(y_{i}-y_{5}\right)$,

\section{Exterior E d ge C on strain ts}

$$
\begin{array}{r}
\sigma_{i}-\sigma_{j}=a_{i j}\left(\theta_{x i}+\theta_{x j}\right)+b_{i j}\left(\theta_{y i}+\theta_{y j}\right), \\
i=1,2,3,4 ; j=2,3,4,1
\end{array}
$$

where $\quad a_{i j}=\frac{1}{2}\left(x_{i}-x_{j}\right), \quad b_{i j}=\frac{1}{2}\left(y_{i}-y_{j}\right)$

Adding the four equations in (7) results in the solution for $\sigma_{5}$,

$$
\sigma_{5}=\frac{1}{4} \sum_{\mathrm{i}=1,4}\left[\sigma_{\mathrm{i}}-\mathrm{a}_{\mathrm{i} 5} \theta_{\mathrm{xi}}-\mathrm{b}_{\mathrm{i} 5} \theta_{\mathrm{yi}}\right]-\mathrm{a} \theta_{\mathrm{x} 5}-\mathrm{b} \theta_{\mathrm{y} 5}
$$

where $\quad a=\frac{1}{8}\left(x_{1}+x_{2}+x_{3}+x_{4}-4 x_{5}\right)$ and

$$
\mathrm{b}=\frac{1}{8}\left(\mathrm{y}_{1}+\mathrm{y}_{2}+\mathrm{y}_{3}+\mathrm{y}_{4}-4 \mathrm{y}_{5}\right) .
$$

Furthermore, a straightforward manipulation of the equations in (7) and (8) yields the matrix equation for the $\theta_{\mathrm{x} 5}$ and $\theta_{\mathrm{y} 5}$ dof's, i.e.,

$$
\left\{\begin{array}{l}
\theta_{\mathrm{x} 5} \\
\theta_{\mathrm{y} 5}
\end{array}\right\}=\frac{2}{\mathrm{~A}_{\mathrm{Q}}}\left[\begin{array}{cc}
\mathrm{b}_{24} & -\mathrm{b}_{13} \\
-\mathrm{a}_{24} & \mathrm{a}_{13}
\end{array}\right]\left[\begin{array}{cc}
-\mathrm{a}_{25} & 0 \\
\mathrm{a}_{13} & -\mathrm{a}_{35} \\
\mathrm{a}_{25} & \mathrm{a}_{24} \\
0 & \mathrm{a}_{35} \\
-\mathrm{b}_{25} & 0 \\
\mathrm{~b}_{13} & -\mathrm{b}_{35} \\
\mathrm{~b}_{25} & \mathrm{~b}_{24} \\
0 & \mathrm{~b}_{35}
\end{array}\right]^{\mathrm{T}}\left[\begin{array}{c}
\theta_{\mathrm{x} 1} \\
\theta_{\mathrm{x} 2} \\
\theta_{\mathrm{x} 3} \\
\theta_{\mathrm{x} 4} \\
\theta_{\mathrm{y} 1} \\
\theta_{\mathrm{y} 2} \\
\theta_{\mathrm{y} 3} \\
\theta_{\mathrm{y} 4}
\end{array}\right\}
$$

where $A_{Q}=2\left(a_{13} b_{24}-a_{24} b_{13}\right)$ is the area of the quadrilateral macro-element. Equations (9) and (10) provide the explicit relations between the dof's at the cross-diagonal node (node 5) and the vertex nodes of the quadrilateral.

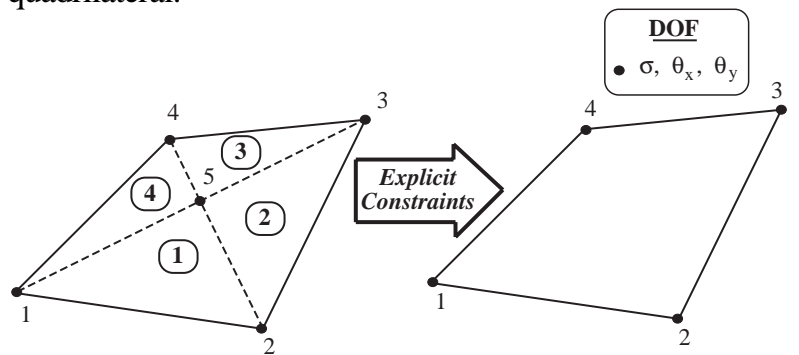

Fig. 3. Reduction of five-node macro-element to fournode quadrilateral via edge constraints.

There are two equivalent approaches of employing the reduction equations (9) and (10) to construct the four-node quadrilateral: (a) The element matrices for the four triangles comprising the five-node macro-element are pre-imposed and multiplied by the appropriate transformation matrices, $\mathbf{T}_{\mathrm{j}}(\mathrm{j}=1,4)$, resulting from (9) and (10). This process is variationally consistent and is commonly employed whenever transformations of dof's are performed, (b) Alternatively, (9) and (10) may be used directly to modify the interpolation functions for each of the four triangles in the five-node macroelement. Using the foremost method, the quadrilateral element equations are as follows:

$$
\mathbf{K}_{\mathrm{Q}}^{\mathrm{e}} \mathbf{d}_{\mathrm{Q}}^{\mathrm{e}}=\mathbf{F}_{\mathrm{Q}}^{\mathrm{e}}
$$

where

$$
\mathbf{K}_{\mathrm{Q}}^{\mathrm{e}}=\sum_{\mathrm{j}=1,4} \mathbf{T}_{\mathrm{j}}^{\mathrm{T}} \mathbf{K}_{\mathrm{j}}^{\mathrm{e}} \mathbf{T}_{\mathrm{j}}, \quad \mathbf{F}_{\mathrm{Q}}^{\mathrm{e}}=\sum_{\mathrm{j}=1,4} \mathbf{T}_{\mathrm{j}}^{\mathrm{T}} \mathbf{F}_{\mathrm{j}}^{\mathrm{e}},
$$

and

$$
\mathbf{d}_{\mathrm{Q}}^{\mathrm{e}}=\left\{\sigma_{\mathrm{i}}, \theta_{\mathrm{xi}}, \theta_{\mathrm{yi}}\right\}^{\mathrm{T}} \quad(\mathrm{i}=1,4),
$$


and where the summation is over the four triangular elements.

It should be noted that static condensation of the interior dof's would result in the same solution as the quadrilateral smoothing solution due to (11), however the systems of equations are in different bases and are therefore not identical. In addition, static condensation is not as computationally efficient as the explicit reduction equations (9) and (10).

\section{Num erical E xam ples}

Two adaptive mesh refinement solutions are carried out to demonstrate the robustness and accuracy of the smoothing method and the computational efficiency of the four-node smoothing element. First, a linearly elastic plate with a small central hole under compression is analyzed to verify the equivalence of the five-node, cross-diagonal pattern macro-element and the four-node quadrilateral, and to demonstrate the computational efficiency of the four-node element. The second numerical solution deals with a built-up aerospace structural component under compression and bending, where shell elements are located in different planes. In this structure, stresses at shell junctions are in general discontinuous, and for this reason, SEA is performed on different domains independently.

All computations are obtained with NASA's COMET-AR (COmputational MEchanics Testbed Adaptive Refinement) FEA code on an IBM RS6000 workstation using nine-node fully integrated Lagrange shell elements. ${ }^{22}$ These shell elements are somewhat stiff in bending, however, they are relatively insensitive to mesh distortion - an aspect that is paramount in adaptive mesh refinement. No special consideration is taken for modeling shell junctions. The smoothing mesh generation is fully automatic and uses the same quadrilateral mesh as that of the finite element models. The FEA stresses are sampled at the $3 \times 3$ Gauss points. In the smoothing mesh, these points are located with an efficient search algorithm. ${ }^{24}$

\section{Error E stim ation and A daptive}

\section{Refinem ent}

To demonstrate robustness of the smoothing element due to element distortion, aspect-ratio tolerances are not set in the adaptive refinement. Instead, the maximum number of refinement meshes is specified.

Adaptive mesh refinement is performed using transition-based refinement strategies with a specified refinement tolerance. Any finite element exhibiting an average element error, $\mathrm{R}_{\text {ave }}$, greater than the specified refinement tolerance is subdivided into nine elements. The average element error is computed as

$$
\mathrm{R}_{\mathrm{ave}}=\frac{\mathrm{E}_{\mathrm{e}}}{\left[\mathrm{U}^{\mathrm{ref}} / \mathrm{N}_{\text {elts }}\right]^{\frac{1}{2}}}
$$

in which $\mathrm{E}_{\mathrm{e}}$ is the element error energy norm given as

$$
\mathrm{E}_{\mathrm{e}}=\left[\frac{1}{2} \int_{\Omega_{\mathrm{e}}}\left(\mathbf{S}^{\mathrm{S}}-\mathbf{S}^{\mathrm{h}}\right)^{\mathrm{T}} \mathbf{C}^{-1}\left(\mathbf{S}^{\mathrm{S}}-\mathbf{S}^{\mathrm{h}}\right) \mathrm{d} \Omega\right]^{\frac{1}{2}} .
$$

The vectors $\mathbf{S}^{\mathrm{h}}$ and $\mathbf{S}^{\mathrm{s}}$ contain, respectively, eight FEA and SEA shell stress components, $\mathrm{N}_{\text {elts }}$ is the number of finite elements, and $\mathbf{C}$ is an $8 \times 8$ constitutive matrix. In (12), $\mathrm{U}^{\text {ref }}$ is the reference strain energy defined as the finite element strain energy corrected by the global error $^{23}$

where

$$
\begin{gathered}
\mathrm{U}^{\mathrm{ref}}=\mathrm{U}^{\mathrm{h}}+\mathrm{E}^{2} \\
\mathrm{E}=\left[\sum_{\mathrm{N}_{\mathrm{elts}}} \mathrm{E}_{\mathrm{e}}^{2} / \mathrm{U}^{\mathrm{s}}\right]^{\frac{1}{2}}
\end{gathered}
$$

with $\mathrm{U}^{\mathrm{h}}$ and $\mathrm{U}^{\mathrm{s}}$ denoting the total strain energies corresponding to the FEA and SEA stress fields, respectively.

\section{$\underline{P \text { late } \mathrm{W} \text { ith } \mathrm{H} \text { Ole }}$}

A two-dimensional elasticity problem is analyzed to demonstrate the robustness and efficiency of SEA in the context of automatic adaptive refinement. A rectangular aluminum plate (thickness $=0.1$ in., $E=10 \mathrm{Msi}, v=$ 0.3 ) is subjected to a tensile uniform displacement of 0.1 in. along an edge parallel to the $y$-axis that is constrained to move only in the $\mathrm{x}$ direction (refer to Fig. 4). The opposite edge is clamped and the edges parallel to the $\mathrm{x}$-axis are free. The problem is particularly challenging because the hole is very small, $\mathrm{d} / \mathrm{w}=0.05$, where $\mathrm{d}$ is the hole diameter. This aspect tends to produce distorted elements around the hole where a stress concentration takes place. Stress singularities at the plate corners, where the displacement boundary conditions are prescribed, provide another challenge for the adaptive refinement. ${ }^{21}$

The initial mesh (Mesh 0) and three consecutive, automatically generated meshes are depicted in Fig. 4. At each refinement step, finite elements of greater distortion and smaller size are generated. This is especially true near the hole and at the plate corners the regions of high stress gradients. By applying SEA 

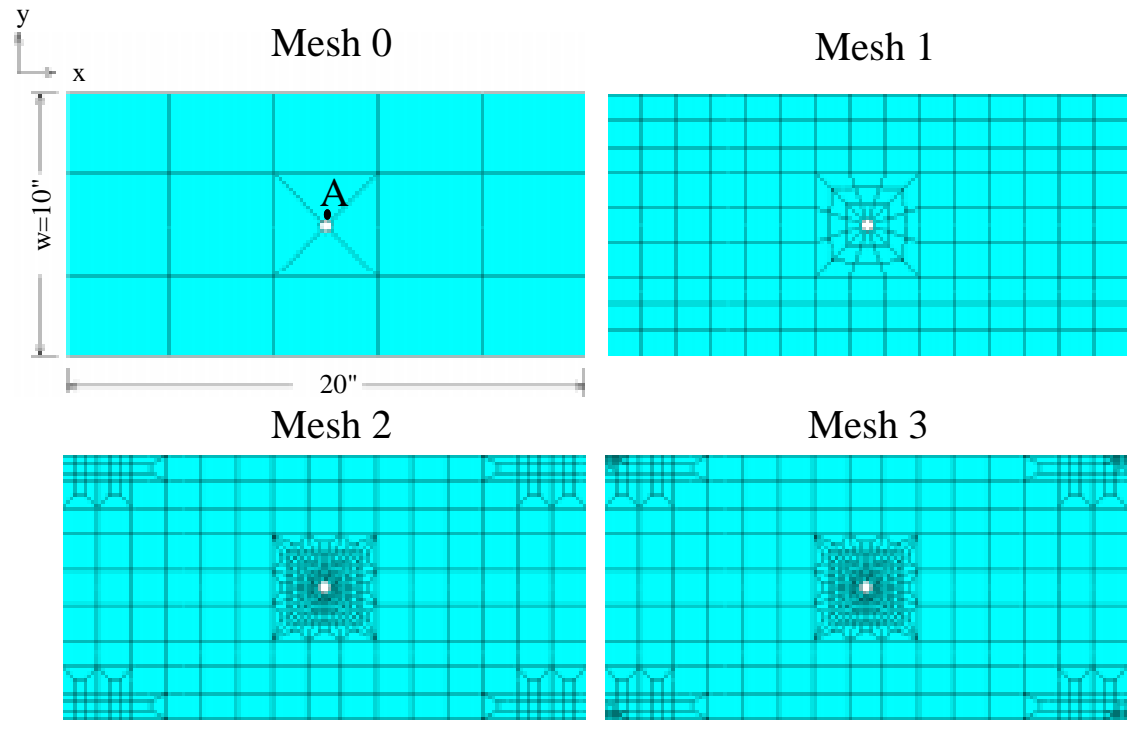

Fig. 4. Plate with hole. Initial (Mesh 0) and three refinement meshes.

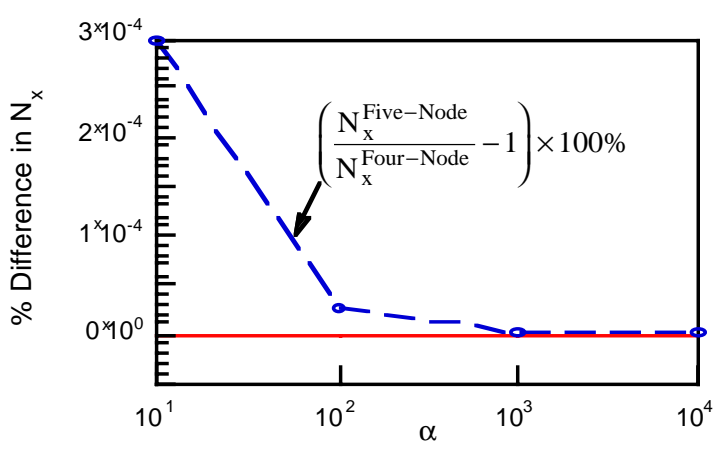

Fig. 5. Percent difference in $\mathrm{N}_{\mathrm{x}}$ at point $A$ due to fiveand four-node quadrilateral SEA solutions.

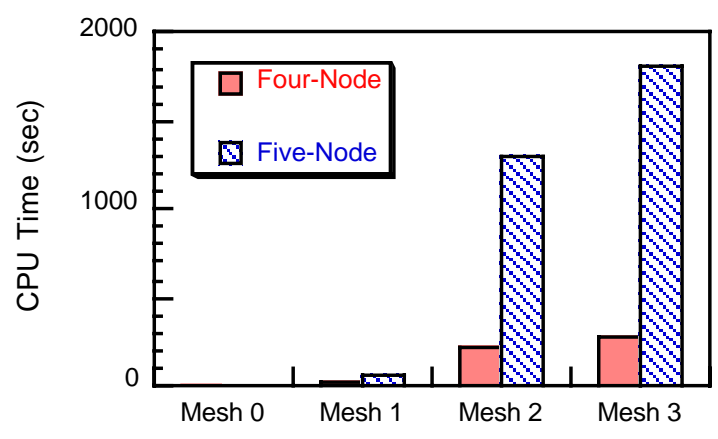

Fig. 6. Comparison of CPU time for SEA with fourand five-node quadrilaterals.

for each mesh, robust stress smoothing and error estimation is achieved. For Mesh 3, possessing 4,476 finite element dof's, a global error norm of less than $1 \%$ is obtained.
In Fig. 5, the equivalence between the five- and fournode quadrilateral smoothing solutions for Mesh 0 is demonstrated. At point A located at the top of the hole perimeter, where the axial stress resultant $\mathrm{N}_{\mathrm{x}}$ is maximum, the solutions approach the same value as the penalty parameter $\alpha$ becomes larger. Apparently, the four-node solution does not suffer from any loss of accuracy as a result of the explicit dof reduction inherent in the element formulation. Even at relatively small values of $\alpha(\alpha=10)$, the two SEA solutions are within $0.0003 \%$. This excellent degree of correlation is typical for the highly stressed regions in the plate.

Having fewer dof's and achieving equivalent results, the four-node smoothing element is significantly more efficient than the original five-node macro-element. This aspect is clearly demonstrated in Fig. 6 which compares CPU time for the two SEA modeling approaches. Evidently, the larger the mesh, the greater the computational savings attained. Thus, for Mesh 3, the SEA computation is reduced by a factor of 6.6 with the use of the four-node smoothing quadrilateral element.

\section{H at-S tiffened Panel}

The hat-stiffened panel is an example of a built-up aerospace structural component. The panel dimensions and boundary conditions in Fig. 7 are shown on the initial (Mesh 0) discretization. The right edge of the panel is subjected to a compressive axial (x-direction) displacement of 0.1 in., including the stiffener edge. The opposite edges of the panel and stiffener are fixed. Along the side edges, symmetric boundary conditions are prescribed. Whereas FEA is carried out with a nine- 


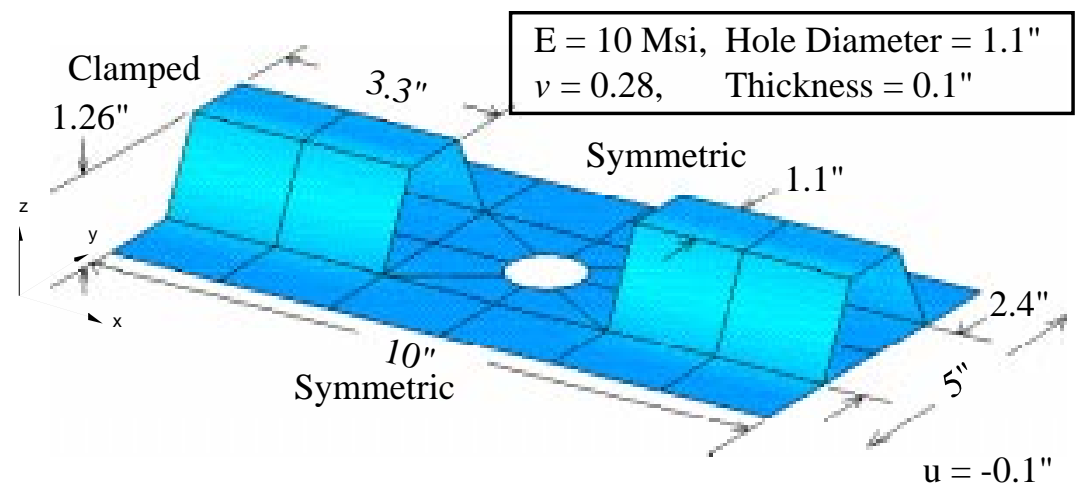

Fig. 7. Hat-stiffened panel.

node Lagrange shell element, SEA is performed using the four-node quadrilateral with the element parameters set at $\alpha=10^{5}$ and $\beta=0$.

From the structural response standpoint, the panel has a number of challenging aspects: (a) the stiffness eccentricity due to the hat stiffener located on only one side of the panel causes local bending, (b) stress concentrations occur along the hole perimeter, and (c) singular stresses develop at the ends of the stiffenerpanel junctions, where stresses are transferred according to the shear-lag mechanism. It must be noted that the initial mesh is very coarse and does not address any of the aforementioned issues.

Knowing that stress fields at the stiffener-panel shell junctions are generally discontinuous, the application of SEA is carried out on separate, independent domains to avoid unphysical smoothing across these junctions. In Fig. 8, the panel modeled with Mesh 0 is broken-up into nine independent smoothing domains. Within each domain, stress resultants are defined to correspond to a convenient coordinate system, and these stress components are smoothed independently. At each refinement step, error estimation is undertaken based on the domain smoothed stresses. The distributions of the average element error (refer to (12)) corresponding to the initial and two adaptive refinement discretizations are shown in Fig. 9.

An examination of specific stress distributions computed via FEA and subsequent SEA for error estimation, further validates the effectiveness and robustness of the SEA stress recovery. The typical stress results are depicted in Figs. 10 and 11, which also display the number of dof's and CPU time for the entire FEA and SEA computations, including model definition. The SEA CPU time includes the smoothing of all eight shell stress components (i.e., three in-plane stress resultants, three bending moments, and two transverse shear stress resultants) for each domain.

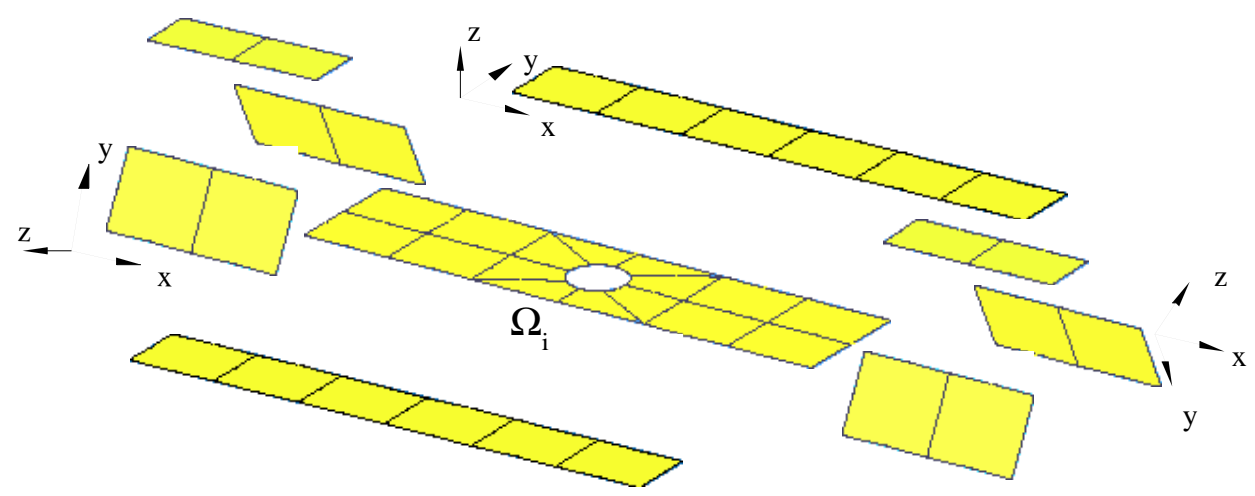

Fig. 8. Hat-stiffened panel. Independent smoothing domains. 


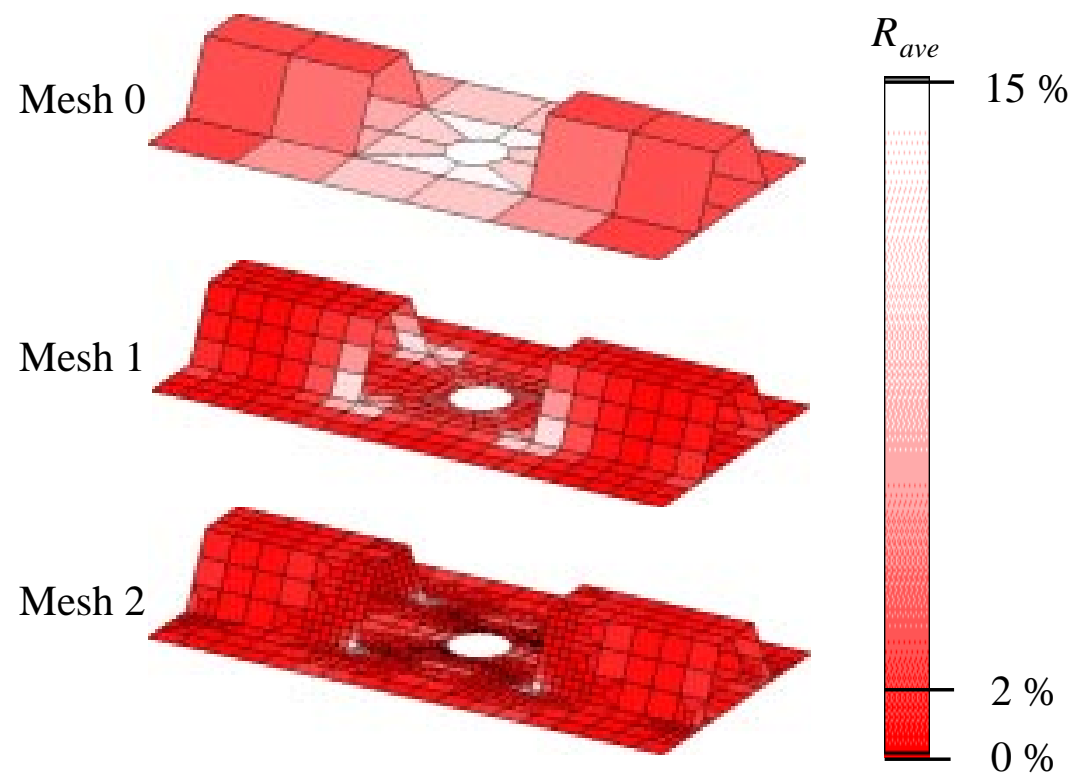

Fig. 9. Hat-stiffened panel mesh. Refinement steps and associated average element error.

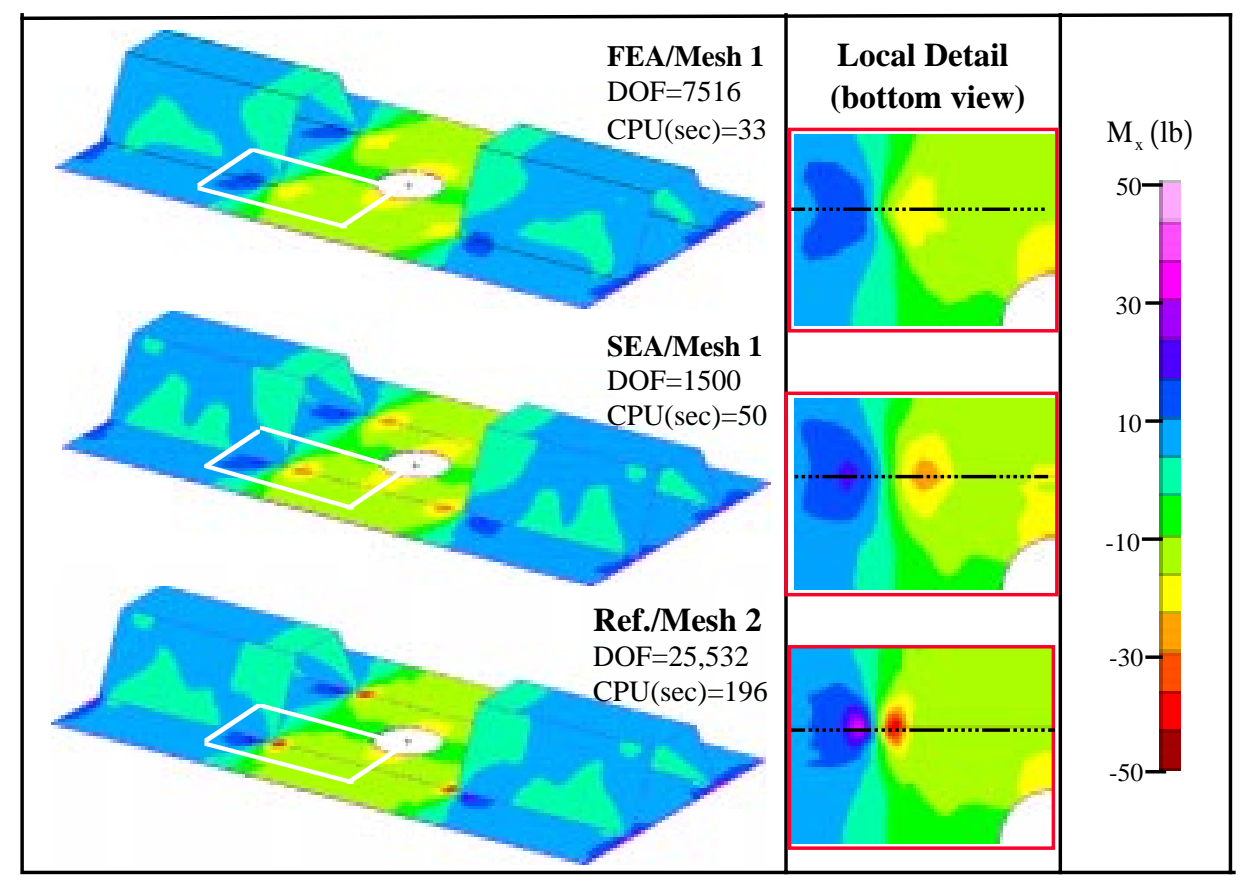

Fig. 10. Hat-stiffened panel. Mistben்ỉ ǵomoment.

Figure 10 shows the $\mathrm{M}_{\mathrm{x}}$ bending moment distribution, where the FEA results for Mesh 1 are averaged at all common element boundaries, enforcing stress continuity at the shell junctions. This type of post-processing is standard in most general-purpose finite element codes. For reference purposes, the results for a highly refined mesh (Mesh 2) are used.
Since the domain-based SEA does not enforce stress continuity across domain junctions, the resulting smoothed $\mathrm{M}_{\mathrm{x}}$ distribution is considerably more accurate than that of the FEA, strongly resembling the reference solution. Observe that SEA-based $\mathrm{M}_{\mathrm{x}}$ distribution is nearly continuous across domains where it is expected, without being enforced (refer to the local details in Fig. 10). Also note that SEA-based $M_{x}$ 


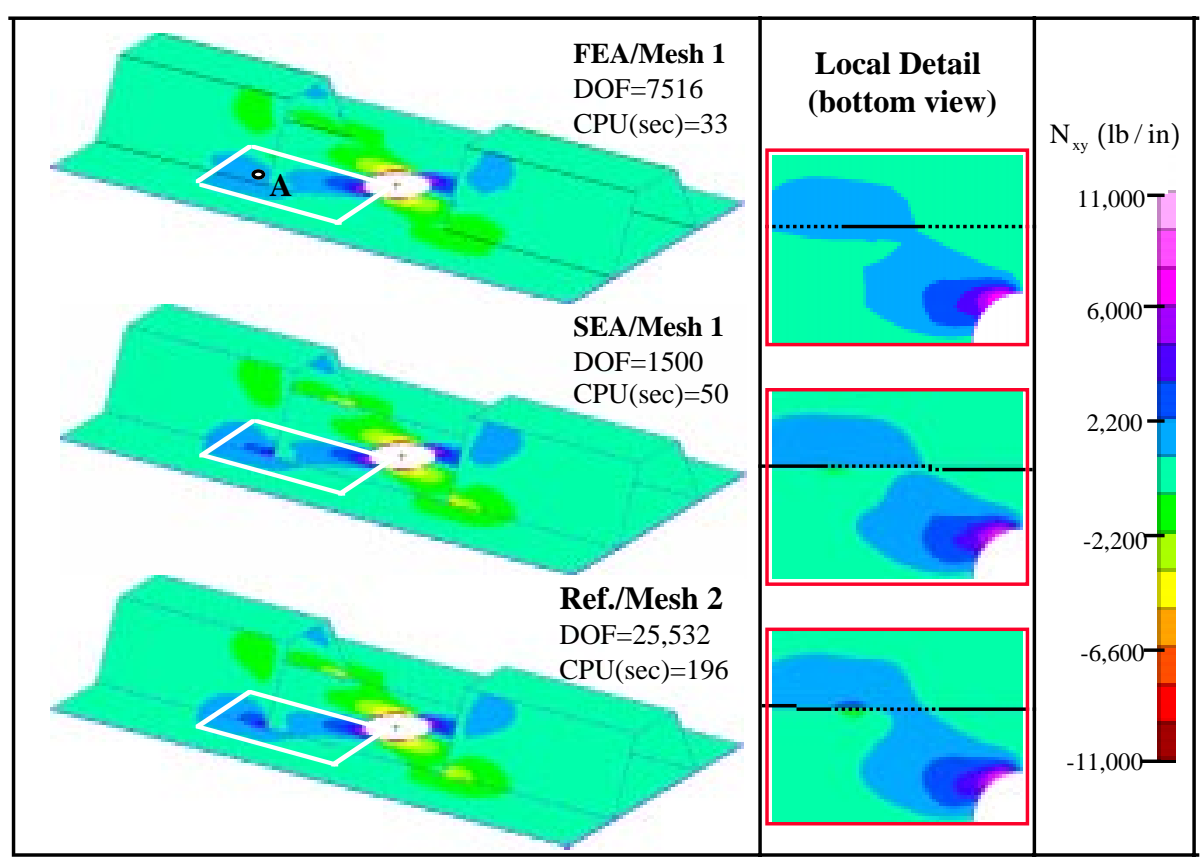

Fig. 11. Hat-stiffened panel. Distribution of $\mathrm{N}_{\mathrm{xy}}$ stress resultant.

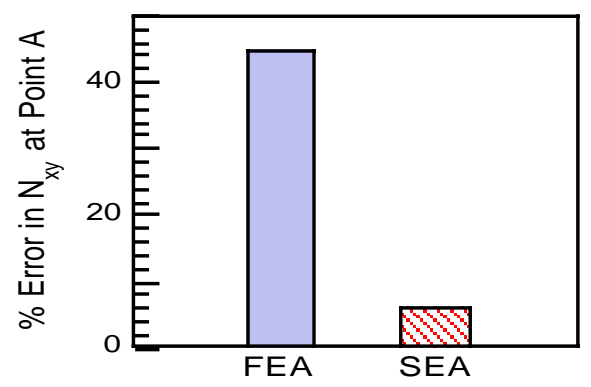

Fig. 12. FEA and SEA (Mesh 1) accuracy comparisons for $\mathrm{N}_{\mathrm{xy}}$ at point $\mathrm{A}$.

'uncovers' the physical stress concentrations that are not particularly evident from the FEA results.

In Fig. 11, the in-plane shear resultant $\mathrm{N}_{\mathrm{xy}}$ distributions for Mesh 1 and Mesh 2 (reference) are presented. These results demonstrate once again the importance of the independent domain-based smoothing for stress post-processing. For example, the $\mathrm{N}_{\mathrm{xy}}$ stress resultant defined in the stiffener which intersects the base panel has a different definition than that in the base panel. Yet, unless special care is taken, these results are commonly averaged at the shell junctions, producing erroneous results at the intersections and surrounding regions. With the domain-based SEA, however, stress continuity is not enforced across the distinct domains and physically meaningful stresses are recovered. Observe the similarities of the SEA and reference distributions.
An example of quantitative stress improvement associated with the SEA recovery is shown in Fig. 12, where, corresponding to Mesh $1, \mathrm{~N}_{\mathrm{xy}}$ is examined in a region of high-stress gradient. It is seen that at point $\mathrm{A}$, which is located close to the edge of the left stiffener, there is a $38 \%$ reduction in the error for $\mathrm{N}_{\mathrm{xy}}$ due to smoothing.

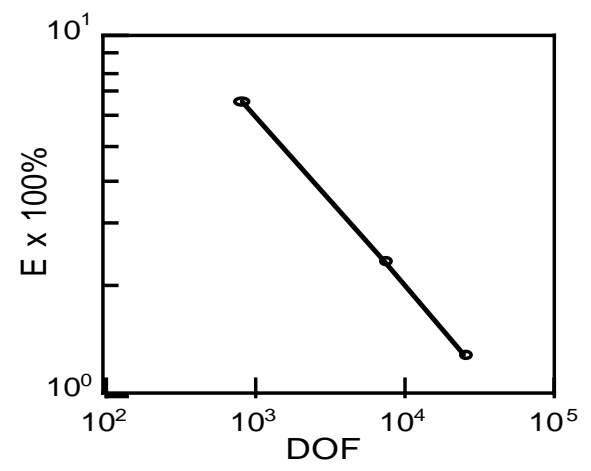

Fig. 13. FEA global percent error in energy norm vs. dof's.

It is evident that SEA-based stress recovery is effective in identifying stress concentrations in relatively coarse FEA models, which allows for more rapid global convergence in adaptive mesh refinement. The convergence of the adaptive refinement process is illustrated in Fig. 13. In the figure, the global FEA percent error in the energy norm (computed according to (14) by adding 
all element contributions) is plotted versus the number of finite element dof's.

\section{$\underline{\mathrm{C} \text { on cluding } \mathrm{R} \text { em arks }}$}

A four-node quadrilateral smoothing element has been developed as a result of an explicit dof reduction solution. The original five-node macro-element formed with four smoothing triangles in a cross-diagonal pattern has been reduced to a four-node quadrilateral by exactly enforcing a set of natural edge-wise constraint equations. The method, smoothing element analysis, provides $\mathrm{C}^{1}$-continuous recovered stress distributions based on the minimization of a penalized-discrete-leastsquares error functional. The error functional involves a discrete least-squares term in which discrete finite element stresses are compared with continuous recovered stresses, a penalty constraint term that enforces $C^{1}$ continuity of the recovered stresses, and a curvaturecontrol term that ensures stability and robustness of the method. The four-node element is shown to provide equivalent results with the original triangular element while significantly decreasing computational cost. The recovered $\mathrm{C}^{1}$-continuous stress distributions have been employed in a posteriori error estimation in NASA's COMET-AR finite element code, thus enabling efficient adaptive mesh refinement solutions, including those for built-up aerospace structures.

\section{$\underline{R \text { eferen ces }}$}

1. Oden, J. T., and Brauchli, H. J., "On the Calculation of Consistent Stress Distributions in Finite Element Approximations," International Journal for Numerical Methods in Engineering, 3,1971, pp. 317- 325.

2. Hinton, E., and Campbell, J. S., "Local and Global Smoothing of Discontinuous Finite Element Functions Using a Least Squares Method," International Journal for Numerical Methods in Engineering, 8, 1974, pp. 461-480.

3. Oden, J. T., and Reddy, J. N., "Note on an Approximate Method for Computing Consistent Conjugate Stresses in Elastic Finite Elements," International Journal for Numerical Methods in Engineering, 6, 1973, pp. 55-61.

4. Barlow, J., "Optimal Stress Locations in Finite Element Models," International Journal for Numerical Methods in Engineering, 10, 1976, pp. 243-251.

5. Wilson, E. L., and Ibrahimbegovic, A., "Use of Incompatible Displacement Modes for the Calculation of Element Stiffnesses or Stresses," Finite Elements in Analysis and Design, 7, 1990, pp. 229- 241.

6. Zienkiewicz, O. C., and Zhu, J. Z., "A Simple

Error Estimator and Adaptive Procedure for Practical Engineering Analysis," International Journal for Numerical Methods in Engineering, 24, 1987, pp. 337-357.

7. Zienkiewicz, O. C., and Zhu, J. Z., "The Superconvergent Patch Recovery and A Posteriori Error Estimates. Part 1: The Recovery Technique," International Journal for Numerical Methods in Engineering, 33, 1992, pp. 1331-1364.

8. Zienkiewicz, O. C., and Zhu, J. Z., "The Superconvergent Patch Recovery (SPR) and Adaptive Finite Element Refinement," Computer Methods in Applied Mechanics and Engineering, 101, 1992, pp. 207-224.

9. Wiberg, N. E., and Abdulwahab, F., "Patch Recovery Based on Superconvergent Derivatives and Equilibrium," International Journal for Numerical Methods in Engineering, 36, 1993, pp. 2703-2724.

10. Belytschko, T., and Blacker, T., "Enhanced Derivative Recovery through Least Square Residual Penalty," Applied Numerical Mathematics, 14(1), 1994, pp. 55-68.

11. Lee, T.O., Park, H.C., and Lee, S.W., "A Superconvergent Stress Recovery Technique with Equilibrium Constraint," International Journal for Numerical Methods in Engineering, 40(7), 1997, pp. 1139-1160.

12. Boroomand, B., and Zienkiewicz, O. C., "Recovery by Equilibrium in Patches (REP)," International Journal for Numerical Methods in Engineering, 40, 1997, pp. 137-164.

13. Boroomand, B., and Zienkiewicz, O. C., "An Improved REP Recovery and the Effectivity Robustness Test," International Journal for Numerical Methods in Engineering, 40, 1997, pp. 3247-3277.

14. Tessler, A., Freese, C., Anastasi, R., Serabian, S., Oplinger, D., and Katz, A., "Least-Squares PenaltyConstraint Finite Element Method for Generating Strain Fields from Moire Fringe Patterns," Proceedings, International Conference on Photomechanics and Speckle Metrology, San Diego, 814, 1987, pp. 314-323.

15. Tessler, A., and Freese, C., "A Global PenaltyConstraint Finite Element Formulation for Effective Strain and Stress Recovery," Proceedings, Computational Mechanics '91, Theory and Applications, Melbourne, Australia, 1991, pp. 1120-1123.

16. Tessler, A., Riggs, H. R., and Macy, S. C., "Application of a Variational Method for 
Computing Smooth Stresses, Stress Gradients, and Error Estimation in Finite Element Analysis," in J. R. Whiteman (eds.), The Mathematics of Finite Elements and Applications, John Wiley \& Sons, Ltd., 1994, pp. 189-198.

17. Tessler, A., Riggs, H. R., and Macy, S. C., “A Variational Method for Finite Element Stress Recovery and Error Estimation," Computer Methods in Applied Mechanics and Engineering, 111, 369-382 (1994); also in Proceedings, 34th Structures, Structural Dynamics, and Materials Conference, La Jolla, CA, 2, 1993, pp. 739-752.

18. Riggs, H. R., and Tessler, A., "Continuous versus Wireframe Variational Smoothing Methods for Finite Element Stress Recovery," Proceedings, Advances in Post and Preprocessing for Finite Element Technology, Computational Structures Technology, Athens, Greece, 1994, pp. 137-144.

19. Tessler, A., and Riggs, H. R., "Accurate Interlaminar Stress Recovery from Finite Element Analysis," NASA Technical Memorandum 109149, 1994.

20. Riggs, H. R., Tessler, A., and Chu, H., " $C^{1}$ Continuous Stress Recovery in Finite Element Analysis," Computer Methods in Applied Mechanics and Engineering, 143(3/4), 1997, pp. 299-316.

21. Tessler, A., Riggs, H. R., Freese, C. E., and Cook, G. M., “An Improved Variational Method for Finite Element Stress Recovery and a Posteriori Error Estimation," Computer Methods in Applied Mechanics and Engineering, to appear, 1998.

22. Moas, E. (Editor), "COMET-AR User's Manual," NASA/CR-97-206284, December 1997.

23. Moas, E., Sues, R. H., Warnaar, D. B., and Zhang, Y., "Advanced Aerospace Structural Analysis. Validation of the COMET-AR Software System," Applied Research Associates Report No. 5923 prepared for Air Force Wright Laboratory, 1995.

24. Moas, E., Aminpour, M. A., Krishnamurthy, T., and Sues, R. H., "Advanced Aerospace Structural Analysis," Air Force Wright Laboratory Report, WL-TR-96-3053, March 1996.

25. Hughes, T. J. R., "The Finite Element Method. Linear Static and Dynamic Finite Element Analysis," Prentice Hall, Englewood Cliffs, NJ, 1987.

26. Tessler, A., and Hughes, T. J. R., "A Three-Node Mindlin Plate Element with Improved Transverse Shear," Computer Methods in Applied Mechanics and Engineering, 50, 1985, pp. 71-101.

27. Tessler, A., "A Priori Identification of Shear Locking and Stiffening in Triangular Mindlin Elements," Computer Methods in Applied Mechanics and Engineering, 53, 1985, pp. 183-200.

28. Tessler, A., and Dong, S. B., "On a Hierarchy of Conforming Timoshenko Beam Elements," Computers and Structures, 14, 1981, pp. 335-344. 